United Nations Educational Scientific and Cultural Organization and

International Atomic Energy Agency

THE ABDUS SALAM INTERNATIONAL CENTRE FOR THEORETICAL PHYSICS

\title{
LIE-THEORETIC GENERATING RELATIONS OF TWO VARIABLE LAGUERRE POLYNOMIALS
}

\author{
Subuhi Khan \\ Department of Mathematics, Aligarh Muslim University, \\ Aligarh-202002, India \\ and \\ The Abdus Salam International Centre for Theoretical Physics, Trieste, Italy \\ and \\ Ghazala Yasmin \\ Department of Mathematics, Aligarh Muslim University, \\ Aligarh-202002, India.
}

\begin{abstract}
Generating relations involving two variable Laguerre polynomials $L_{n}(x, y)$ are derived. The process involves the construction of a three dimensional Lie algebra isomorphic to special linear algebra $s l(2)$ with the help of Weisner's method by giving suitable interpretations to the index $n$ of the polynomials $L_{n}(x, y)$.
\end{abstract}

MIRAMARE - TRIESTE

July 2002 


\section{INTRODUCTION}

Recently Dattoli and Torre [4,5] introduced and discussed a theory of two variable Laguerre polynomials (TVLP). The reason of interest for this family of Laguerre polynomials is due to their intrinsic mathematical importance and to the fact that these polynomials are shown to be natural solutions of a particular set of partial differential equations, which often appears in the treatment of radiation physics problems such as the electromagnetic wave propagation and quantum beam life-time in storage rings, see Wrülich[13].

The TVLP $L_{n}(x, y)$ are specified by series ([2]; p.121(69))

$$
L_{n}(x, y)=\sum_{r=0}^{n} \frac{(-1)^{r} n ! x^{r} y^{n-r}}{(r !)^{2}(n-r) !}
$$

and the generating function for $L_{n}(x, y)$ is given by ([4]; p.3(10)).

$$
\sum_{n=0}^{\infty} L_{n}(x, y) t^{n}=\frac{1}{(1-y t)} \exp \left(-\frac{x t}{(1-y t)}\right), \quad|y t|<1 .
$$

These polynomials satisfy the following differential and pure recurrence relations:

$$
\begin{aligned}
\frac{\partial}{\partial x} L_{n}(x, y) & =\frac{n}{x} L_{n}(x, y)-\frac{n y}{x} L_{n-1}(x, y) \\
\frac{\partial}{\partial y} L_{n}(x, y) & =n L_{n-1}(x, y) \\
L_{n+1}(x, y) & =\frac{1}{(n+1)}\left(((2 n+1) y-x) L_{n}(x, y)-n y^{2} L_{n-1}(x, y)\right)
\end{aligned}
$$

The TVLP $L_{n}(x, y)$ are linked to the Laguerre polynomials $L_{n}(x)$ [1] by the relation ([5];p.22(10b))

$$
L_{n}(x, y)=y^{n} L_{n}\left(\frac{x}{y}\right), L_{n}(x, 0)=(-1)^{n} \frac{x^{n}}{n !} .
$$

The differential equation satisfied by $L_{n}(x, y)$ is

$$
\left(x \frac{d^{2}}{d x^{2}}+\left(1-\frac{x}{y}\right) \frac{d}{d x}+\frac{n}{y}\right) L_{n}(x, y)=0 .
$$

Very recently Pathan et al. [9,10] obtained generating relations involving two and three variables generalized Hermite polynomials $H_{n}(x, y)$ and $H_{n}(x, y, z)$ [3] using the Lie-theoretic method. In the present paper we utilize Weisner's [12] group-theoretic method for obtaining generating relations in the case of TVLP $L_{n}(x, y)$ by giving suitable interpretations to the index $n$.

It is remarked that another, more general, approach to obtain Laguerre polynomials of many variables (and other polynomials as well, such as Hermite, Charlier) using a chaos decomposition 
on spaces of Fock type can be found in [6] and the references therein. However, the appproach in this paper is more direct and appropriate for obtaining generating relations.

The process involves the construction of a three dimensional Lie algebra isomorphic to special linear algebra $\operatorname{sl}(2)$, the Lie algebra of $S L(2)$ ([8]; p.7). The $2 \times 2$ complex special linear group $S L(2)$ is the abstract matrix group of all $2 \times 2$ nonsingular matrices

$$
g=\left(\begin{array}{ll}
a & b \\
c & d
\end{array}\right), \quad a, b, c, d \in \mathbb{C},
$$

such that det $g=1 . S L(2)$ is a three dimensional local Lie group. The elements

$$
\mathcal{J}^{+}=\left(\begin{array}{rr}
0 & -1 \\
0 & 0
\end{array}\right), \quad \mathcal{J}^{-}=\left(\begin{array}{rr}
0 & 0 \\
-1 & 0
\end{array}\right), \quad \mathcal{J}^{3}=\left(\begin{array}{cc}
\frac{1}{2} & 0 \\
0 & -\frac{1}{2}
\end{array}\right),
$$

satisfying the commutation relations

$$
\left[\mathcal{J}^{3}, \mathcal{J}^{ \pm}\right]= \pm \mathcal{J}^{ \pm}, \quad\left[\mathcal{J}^{+}, \mathcal{J}^{-}\right]=2 \mathcal{J}^{3}
$$

form a basis for $s l(2)$.

\section{GROUP-THEORETIC METHOD}

On replacing $\frac{d}{d x}$ by $\frac{\partial}{\partial x}$ and $n$ by $z \frac{\partial}{\partial z}$ in differential equation (1.5), we construct a partial differential equation

$$
\left(x \frac{\partial^{2}}{\partial x^{2}}+\left(1-\frac{x}{y}\right) \frac{\partial}{\partial x}+\frac{z}{y} \frac{\partial}{\partial z}\right) f(x, y ; z)=0 .
$$

Thus $f(x, y ; z)=L_{n}(x, y) z^{n}$ is a solution of (2.1) since $L_{n}(x, y)$ is a solution of (1.5).

First, we consider the following first order linear partial differential operators

$$
\begin{aligned}
J^{3} & =z \frac{\partial}{\partial z}+\frac{1}{2} \\
J^{+} & =x y z \frac{\partial}{\partial x}+y z^{2} \frac{\partial}{\partial z}+(y-x) z \\
J^{-} & =\frac{x}{y z} \frac{\partial}{\partial x}-\frac{1}{y} \frac{\partial}{\partial z}
\end{aligned}
$$

such that 


$$
\begin{aligned}
J^{3}\left[L_{n}(x, y) z^{n}\right] & =\left(n+\frac{1}{2}\right) L_{n}(x, y) z^{n}, \\
J^{+}\left[L_{n}(x, y) z^{n}\right] & =(n+1) L_{n+1}(x, y) z^{n+1}, \\
J^{-}\left[L_{n}(x, y) z^{n}\right] & =-n L_{n-1}(x, y) z^{n-1} .
\end{aligned}
$$

We note that these operators satisfy the commutation relations

$$
\left[J^{3}, J^{ \pm}\right]= \pm J^{ \pm},\left[J^{+}, J^{-}\right]=2 J^{3}
$$

where $[A, B]=A B-B A$.

The above commutation relations show that the set of $J$-operators $\left\{J^{3}, J^{+}, J^{-}\right\}$generate a three dimensional Lie algebra isomorphic to $\operatorname{sl}(2)$, the Lie algebra of $S L(2)$.

In terms of the $J$-operators, we introduce the Casimir operator ([8]; p.32)

$$
\begin{aligned}
\mathcal{C} & =J^{+} J^{-}+J^{3} J^{3}-J^{3} \\
& =x\left(x \frac{\partial^{2}}{\partial x^{2}}+\left(1-\frac{x}{y}\right) \frac{\partial}{\partial x}+\frac{z}{y} \frac{\partial}{\partial z}\right)-\frac{1}{4} .
\end{aligned}
$$

It is easy to verify that $\mathcal{C}$ commutes with $J^{3}, J^{+}$and $J^{-}$, that is

$$
\left[\mathcal{C}, J^{3}\right]=\left[\mathcal{C}, J^{ \pm}\right]=0
$$

Equation (2.4) enables us to write (2.1) as

$$
\mathcal{C} f(x, y ; z)=-\frac{1}{4} f(x, y ; z) .
$$

Now we proceed to compute the multiplier representation $[T(g) f](x, y ; z), \quad g \in S L(2)$, induced by the $J$-operators (2.2). A simple computation using ([11];p.320 (Theorem 7)) and (2.2) gives

$$
\begin{gathered}
{\left[T\left(\exp \left(b^{\prime} \mathcal{J}^{+}\right)\right) f\right](x, y ; z)=\left(1-b^{\prime} y z\right)^{-1} \exp \left(\frac{-b^{\prime} x z}{\left(1-b^{\prime} y z\right)}\right)} \\
. f\left(\frac{x}{\left(1-b^{\prime} y z\right)}, y ; \frac{z}{\left(1-b^{\prime} y z\right)}\right),\left|b^{\prime} y z\right|<1, \\
{\left[T\left(\exp \left(c^{\prime} \mathcal{J}^{-}\right)\right) f\right](x, y ; z)=f\left(\frac{x}{\left(1-\frac{c^{\prime}}{y z}\right)}, y ; z\left(1-\frac{c^{\prime}}{y z}\right)\right),\left|\frac{c^{\prime}}{y z}\right|<1,} \\
{\left[T\left(\exp \left(\tau^{\prime} \mathcal{J}^{3}\right)\right) f\right](x, y ; z)=\exp \left(\frac{\tau^{\prime}}{2}\right) f\left(x, y ; z \exp \left(\tau^{\prime}\right)\right),}
\end{gathered}
$$

defined for $\left|b^{\prime}\right|,\left|c^{\prime}\right|$ and $\left|\tau^{\prime}\right|$ sufficiently small. 
If $g \in S L(2)$ and $d \neq 0$, it is a straightforward computation to show that

$$
g=\exp \left(b^{\prime} \mathcal{J}^{+}\right) \exp \left(c^{\prime} \mathcal{J}^{-}\right) \exp \left(\tau^{\prime} \mathcal{J}^{3}\right)
$$

where $b^{\prime}=-\frac{b}{d}, c^{\prime}=-c d, \exp \left(\frac{\tau^{\prime}}{2}\right)=\frac{1}{d}$ and $a d-b c=1$.

Hence the operator $T(g)$ is given by

$$
\begin{aligned}
& {[T(g) f](x, y ; z)=(d+b y z)^{-1} \exp \left(\frac{b x z}{(d+b y z)}\right) } \\
& . f\left(\frac{x y z}{(d+b y z)(c+a y z)}, y ; \frac{(c+a y z)}{y(d+b y z)}\right), \\
& \max \left(\left|\frac{b y z}{d}\right|,\left|\frac{c}{a y z}\right|\right)<1,|\arg (d)|<\pi .
\end{aligned}
$$

\section{GENERATING RELATIONS}

To accomplish our job of obtaining generating relations, we search for the function $f(x, y ; z)$ which satisfies (2.6). Consider the case when $f(x, y ; z)$ is a common eigenfunction of $\mathcal{C}$ and $J^{3}$, that is, let $f(x, y ; z)$ be a solution of the simultaneous equations

$$
\begin{gathered}
\mathcal{C} f(x, y ; z)=-\frac{1}{4} f(x, y ; z), \\
J^{3} f(x, y ; z)=\left(n+\frac{1}{2}\right) f(x, y ; z),
\end{gathered}
$$

which may be rewritten as

$$
\begin{gathered}
\left(x \frac{\partial^{2}}{\partial x^{2}}+\left(1-\frac{x}{y}\right) \frac{\partial}{\partial x}+\frac{z}{y} \frac{\partial}{\partial z}\right) f(x, y ; z)=0 \\
\left(z \frac{\partial}{\partial z}-n\right) f(x, y ; z)=0
\end{gathered}
$$

Equations (3.2) yield

$$
f(x, y ; z)=L_{n}(x, y) z^{n}
$$

so that, we have

$$
\begin{aligned}
{[T(g) f](x, y ; z)=} & (d+b y z)^{-n-1}\left(\frac{(c+a y z)}{y}\right)^{n} \exp \left(\frac{b x z}{(d+b y z)}\right) \\
& \cdot L_{n}\left(\frac{x y z}{(d+b y z)(c+a y z)}, y\right),
\end{aligned}
$$

satisfying the relation

$$
\mathcal{C}[T(g) f](x, y ; z)=-\frac{1}{4}[T(g) f](x, y ; z)
$$


If $n$ is a non-negative integer, (3.3) has an expansion of the form

$$
[T(g) f](x, y ; z)=\sum_{k=0}^{\infty} A_{k n}(g) L_{k}(x, y) z^{k}
$$

which simplifies to the identity

$$
\begin{gathered}
(d+b y z)^{-n-1}\left(\frac{(c+a y z)}{y}\right)^{n} \exp \left(\frac{b x z}{(d+b y z)}\right) L_{n}\left(\frac{x y z}{(d+b y z)(c+a y z)}, y\right) \\
=\sum_{k=0}^{\infty} A_{k n}(g) L_{k}(x, y) z^{k}
\end{gathered}
$$

To determine $A_{k n}(g)$, we set $x=0$ and $y=1$ in (3.4), and thus we have

$$
\begin{gathered}
A_{k n}(g)=\sum_{r=0}^{n} \frac{(-n)_{r}(n-r+1)_{k-n+r}}{(k-n+r) ! r !}(-b)^{k-n+r}(-c)^{r} d^{-n-k-1}, \\
k, n \geq 0
\end{gathered}
$$

Substituting (3.5) into (3.4) and simplifying, we obtain the generating relation

$$
\begin{gathered}
(a d)^{n}\left(1+\frac{b y z}{d}\right)^{-n-1}\left(1+\frac{c}{a y z}\right)^{n} \exp \left(\frac{b x z}{(d+b y z)}\right) L_{n}\left(\frac{x y z}{(d+b y z)(c+a y z)}, y\right) \\
=\sum_{k=0}^{\infty} \sum_{r=0}^{n} \frac{(-n)_{r}(n-r+1)_{k}}{k ! r !}\left(\frac{-b z}{d}\right)^{k}\left(\frac{-c d}{z}\right)^{r} L_{n+k-r}(x, y) .
\end{gathered}
$$

We consider some special cases of (3.6).

I. Taking $a=c=d=1, b=0$ and replacing $z$ by $-\frac{1}{t}$, in (3.6), we obtain

$$
\left(1-\frac{t}{y}\right)^{n} L_{n}\left(\frac{x}{\left(1-\frac{t}{y}\right)}, y\right)=\sum_{r=0}^{n} \frac{(-n)_{r}}{r !} L_{n-r}(x, y) t^{r}
$$

which for $y=1$ reduces to a special case of ([11]; p.331(31)) (with $\alpha=0$ )

$$
(1-t)^{n} L_{n}\left(\frac{x}{(1-t)}\right)=\sum_{r=0}^{n} \frac{(-n)_{r}}{r !} L_{n-r}(x) t^{r} .
$$

II. Taking $a=b=d=1, c=0$ and replacing $z$ by $-t$, in (3.6), we obtain

$$
(1-y t)^{-n-1} \exp \left(\frac{-x t}{(1-y t)}\right) L_{n}\left(\frac{x}{(1-y t)}, y\right)=\sum_{k=0}^{\infty} \frac{(n+1)_{k}}{k !} L_{n+k}(x, y) t^{k}
$$

which for $y=1$ reduces to ([7]; p.20(12))

$$
(1-t)^{-n-1} \exp \left(\frac{-x t}{(1-t)}\right) L_{n}\left(\frac{x}{(1-t)}\right)=\sum_{k=0}^{\infty} \frac{(n+1)_{k}}{k !} L_{n+k}(x) t^{k}
$$


III. Taking $a=d=1$ and $b c \neq 0$, without any loss of generality we can choose $b z=-t_{1}$ and $\frac{c}{z}=-t_{2}$ in (3.6) and we obtain

$$
\begin{gathered}
\left(1-y t_{1}\right)^{-n-1}\left(1-\frac{t_{2}}{y}\right)^{n} \exp \left(\frac{-x t_{1}}{\left(1-y t_{1}\right)}\right) L_{n}\left(\frac{x}{\left(1-y t_{1}\right)\left(1-\frac{t_{2}}{y}\right)}, y\right) \\
=\sum_{k=0}^{\infty} \sum_{r=0}^{n} \frac{(-n)_{r}(n-r+1)_{k}}{k ! r !} t_{1}^{k} t_{2}^{r} L_{n+k-r}(x, y),
\end{gathered}
$$

which for $y=1$ yields

$$
\begin{gathered}
\left(1-t_{1}\right)^{-n-1}\left(1-t_{2}\right)^{n} \exp \left(\frac{-x t_{1}}{\left(1-t_{1}\right)}\right) L_{n}\left(\frac{x}{\left(1-t_{1}\right)\left(1-t_{2}\right)}\right) \\
=\sum_{k=0}^{\infty} \sum_{r=0}^{n} \frac{(-n)_{r}(n-r+1)_{k}}{k ! r !} t_{1}^{k} t_{2}^{r} L_{n+k-r}(x) .
\end{gathered}
$$

Further taking $t_{1}=t_{2}=t$ in (3.10), we get

$$
\begin{aligned}
(1-t)^{-1} \exp \left(\frac{-x t}{(1-t)}\right) L_{n}\left(\frac{x}{(1-t)^{2}}\right) \\
=\sum_{k=0}^{\infty} \sum_{r=0}^{n} \frac{(-n)_{r}(n-r+1)_{k}}{k ! r !} t^{k+r} L_{n+k-r}(x)
\end{aligned}
$$

Acknowledgements: The authors are thankful to the referee for his valuable suggestions which improved the presentation of the paper. The first author is indebted to Prof. Gregory F. Lawler for useful discussions. She is also thankful to the Abdus Salam International Centre for Theoretical Physics (ICTP), Trieste, Italy, where part of this work has been done, for hospitality.

\section{References}

[1] L. C. Andrews: Special Functions for Engineers and Applied Mathematicians,Macmillan Publishing Company, New York, 1985.

[2] G. Dattoli: Generalized polynomials, operational identities and their applications, Journal of Computational and Applied Mathematics 118 (2000),111-123.

[3] G. Dattoli, C. Chiccoli, S. Lorenzutta, G. Maino and A. Torre: Generalized Bessel functions and generalized Hermite polynomials, J.Math.Anal.Appl. 178 (1993), 509-516.

[4] G. Dattoli and A. Torre: Operational methods and two variable Laguerre polynomials, Acc. Sc. Torino-Atti Sc.Fis. 132(1998), 1-7.

[5] G. Dattoli and A. Torre: Exponential operators, quasi-monomials and generalized polynomials, Radiation Physics and Chemistry 57(2000), 21-26. 
[6] Y. G. Kondratiev, J. L. Da Silva, L. Streit and G. F. Us: Analysis on Poisson and Gamma spaces, Infinite Dimensional Analysis, Quantum Probability and Related Topics 1(1) (1998), 91-117.

[7] E. B. McBride: Obtaining Generating Functions, Springer-Verlag, New York, Heidelberg and Berlin, 1971.

[8] W. Jr. Miller: Lie Theory and Special Functions, Academic Press, New York and London, 1968.

[9] M. A. Pathan, Subuhi Khan and Ghazala Yasmin; Lie theory and two variables generalized Hermite polynomials, (Appearing in Italian Journal of Pure and Applied Mathematics, No.16).

[10] M. A. Pathan, Subuhi Khan and Ghazala Yasmin; Representation of a Lie algebra $\mathcal{G}(0,1)$ and three variables generalized Hermite polynomials, $H_{n}(x, y, z)$, (Appearing in Integral Transforms and Special Functions, 2001-2002).

[11] H. M. Srivastava and H. L. Manocha: A Treatise on Generating Functions, Ellis Horwood Limited, Chichester, 1984.

[12] L. Weisner: Group-theoretic origin of certain generating functions, Pacific J.Math., 5 (1955), 1033-1039.

[13] A. Wrülich: Beam-Life Time in Storage Rings. CERN Accelerator School, 1992. 